\title{
Quantifying the effect of metal-rich precipitates on minority carrier diffusion length in multicrystalline silicon using synchrotron-based spectrally resolved x-ray beam-induced current
}

\author{
T. Buonassisi, A. A. Istratov, ${ }^{\text {a) }}$ and M. D. Pickett \\ Department of Materials Science and Engineering, University of California, Berkeley and Materials Science \\ Division, Lawrence Berkeley National Laboratory, Berkeley, California 94720 \\ M. A. Marcus \\ Advanced Light Source, Lawrence Berkeley National Laboratory, Berkeley, California 94720 \\ G. Hahn \\ University of Konstanz, Department of Physics, 78457 Konstanz, Germany
}

S. Riepe, J. Isenberg, W. Warta, and G. Willeke

Fraunhofer Institute for Solar Energy Systems, Heidenhofstrasse 2, D-79110 Freiburg, Germany

T. F. Ciszek ${ }^{\text {b) }}$

National Renewable Energy Laboratory, Golden, Colorado

\section{E. R. Weber}

Department of Materials Science and Engineering, University of California, Berkeley and Materials Science Division, Lawrence Berkeley National Laboratory, Berkeley, California 94720

(Received 29 April 2005; accepted 2 June 2005; published online 19 July 2005)

\begin{abstract}
Synchrotron-based, spectrally resolved x-ray beam-induced current (SR-XBIC) is introduced as a technique to locally measure the minority carrier diffusion length in semiconductor devices. Equivalence with well-established diffusion length measurement techniques is demonstrated. The strength of SR-XBIC is that it can be combined in situ with other synchrotron-based analytical techniques, such as x-ray fluorescence microscopy ( $\mu$-XRF) and x-ray absorption microspectroscopy ( $\mu$-XAS), yielding information about the distribution, elemental composition, chemical nature, and effect on minority carrier diffusion length of individual transition metal species in multicrystalline silicon. SR-XBIC, $\mu$-XRF, and $\mu$-XAS measurements were performed on intentionally contaminated multicrystalline silicon, revealing a strong correlation between local concentrations of copper and nickel silicide precipitates and a decrease of minority carrier diffusion length. In addition, the reduction of minority carrier diffusion length due to submicron-sized $\mathrm{Cu}_{3} \mathrm{Si}$ and $\mathrm{NiSi}_{2}$ precipitates could be decoupled from the influence of homogeneously distributed nanoprecipitates and point defects. (C) 2005 American Institute of Physics.
\end{abstract}

[DOI: $10.1063 / 1.1997274]$

Konstanzer Online-Publikations-System (KOPS)

URL: http://nbn-resolving.de/urn:nbn:de:bsz:352-2-1i8p164s34ew87

Over the course of the past decade, a suite of synchrotron-based analytical techniques has been developed to study efficiency-limiting, impurity-related defects in solar cell grade multicrystalline silicon (mc-Si). Initially, McHugo, Thompson et al. ${ }^{1,2}$ demonstrated the potential of $\mathrm{x}$-ray fluorescence microscopy $(\mu-\mathrm{XRF})$ to locate and characterize the elemental composition of metal-rich nanoprecipitates within mc-Si solar cells. McHugo et al. ${ }^{3}$ subsequently demonstrated the utility of x-ray absorption microspectroscopy ( $\mu$-XAS) to identify the chemical states of these particles. These two techniques were later developed, with higher flux and submicron spot size, to detect a single iron silicide nanoprecipitate of radius $16 \pm 3 \mathrm{~nm}$ within $1 \mathrm{~s}$, and to characterize its chemical state in minutes. ${ }^{4}$ However, with only $\mu$-XRF and $\mu$-XAS, no direct correlation between the presence of metals and device performance can be made.

Recently, Hieslmair et al. ${ }^{5}$ and Vyvenko et al. ${ }^{6}$ demonstrated the potential of $\mathrm{x}$-ray beam induced current (XBIC) to

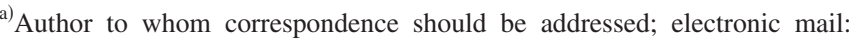
istratov@berkeley.edu

${ }^{b}$ Currently at: Siliconsultant, P. O. Box 1453, Evergreen, Colorado 80437.
}

map the recombination activity, in situ at the $\mu$-XRF/XAS beamline. ${ }^{6}$ The physical principle of XBIC is similar to light/ laser beam induced current (LBIC), in that incident photons generate minority carriers which are collected by a Schottky diode or $p n$ junction; however, $\mathrm{x}$-rays are used instead of visible light. XBIC was successfully combined with $\mu$ $\mathrm{XRF} / \mu$-XAS on several occasions to demonstrate the recombination activity of iron ${ }^{4}$ and copper ${ }^{7}$ related nanodefects in mc-Si, and to correlate results with LBIC maps. ${ }^{8}$ However, with XBIC alone, it is difficult to quantitatively measure the effect of metals on the minority carrier diffusion length.

This is now easily achieved via the technique we propose to call spectrally resolved $\mathrm{x}$-ray beam-induced current (SR-XBIC). The theory behind SR-XBIC is very similar to the spectrally resolved laser beam induced current (SRLBIC) technique: ${ }^{9}$ current collected by the junction is measured for $\mathrm{x}$-rays with different penetration depths, and the current collection efficiency (fraction of photogenerated carriers collected by the $p n$ junction or Schottky diode) is calculated from the known flux and the number of electron-hole pairs generated by each $\mathrm{x}$-ray photon. (X-ray photon flux is 


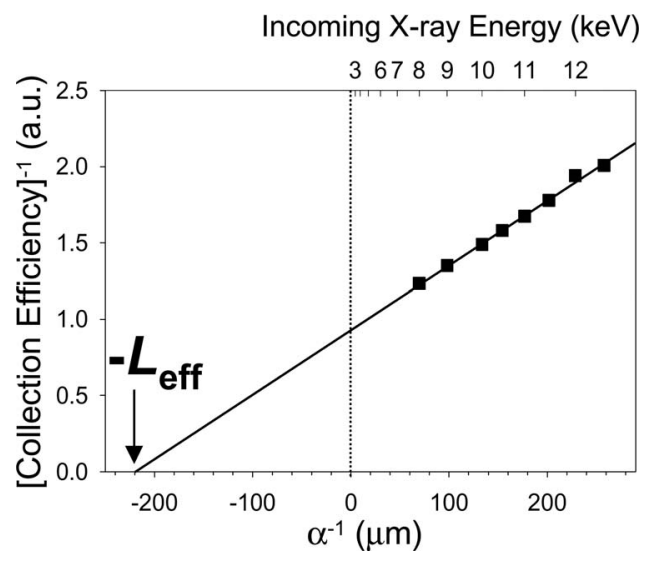

FIG. 1. SR-XBIC determines a diffusion length of $219.9 \pm 7 \mu \mathrm{m}$ at a single point on a monocrystalline $\mathrm{Si}$ sample, which was pre-characterized by the SPV technique and determined to have a diffusion length of $220 \mu \mathrm{m}$. A monochromator was used to vary the energy of the incoming x-rays for each data point on the graph, thus changing the penetration depth according Eq. (1).

determined using a calibrated ion chamber positioned before the sample. One electron-hole pair is generated for each 3.61 $\mathrm{eV}$ of $\mathrm{x}$-ray energy, e.g., a $7 \mathrm{keV}$ x-ray would produce $\sim 1940$ minority carriers.) Then, following the usual methods, ${ }^{10,11}$ a graph is produced comparing $\mathrm{CCE}^{-1}$ to $\alpha^{-1}$, where CCE is the charge collection efficiency, $\alpha$ is the optical absorption coefficient, and $\alpha^{-1}$ is the attenuation (or absorption) length. For $\alpha^{-1}$ much greater than the depletion width but small enough for back surface recombination not to significantly influence CCE, an effective diffusion length $L_{\text {eff }}$ can be extracted, as shown in Fig. 1.

The only major differences between SR-XBIC and SRLBIC arise from the distinct natures of the excitation sources (hard x-rays versus visible laser light). A fit to available x-ray absorption data tables ${ }^{12}$ indicates that when the incoming x-ray energy $E$ is between 3 and $13 \mathrm{keV}$, specifically for silicon,

$$
\alpha^{-1}(\mu \mathrm{m}) \approx 0.1729 \cdot E(\mathrm{keV})^{2.8881} \text {. }
$$

Within this energy range, $\alpha^{-1}$ is between 4 and $290 \mu \mathrm{m}$, which includes typical values for minority carrier diffusion lengths in mc-Si. Fortuitously, this energy range is available at hard x-ray microprobe beamlines, which are usually designed to scan over the $K$ edges of the $3 d$ transition elements.

To test the quantitative accuracy of SR-XBIC, comparisons with several other diffusion length measurement techniques were performed. Firstly a surface photovoltage (SPV, Ref. 13) calibration standard with $500 \mu \mathrm{m}$ thickness and $220 \mu \mathrm{m}$ diffusion length was measured. SR-XBIC consistently revealed diffusion lengths between 215 and $232 \mu \mathrm{m}$ (e.g., Fig. 1), accurate within experimental error. Since SPV measures the minority carrier diffusion length at low injection levels, ${ }^{14}$ this result suggests that SR-XBIC also measures under low injection conditions, i.e., when $\Delta n \ll p_{0}$. This conclusion is further substantiated by SR-XBIC measurements on samples of $n$ - and $p$-type silicon with strong injection dependences. It must be noted that changes in photon flux and sample doping concentration may alter the condition $\Delta n \ll p_{0}$, and thus, the injection conditions. In particular, the $\mathrm{x}$-ray flux may vary by orders of magnitude, depending on the synchrotron beamline, focusing optics, and aperture settings. All experiments in this letter were performed at the
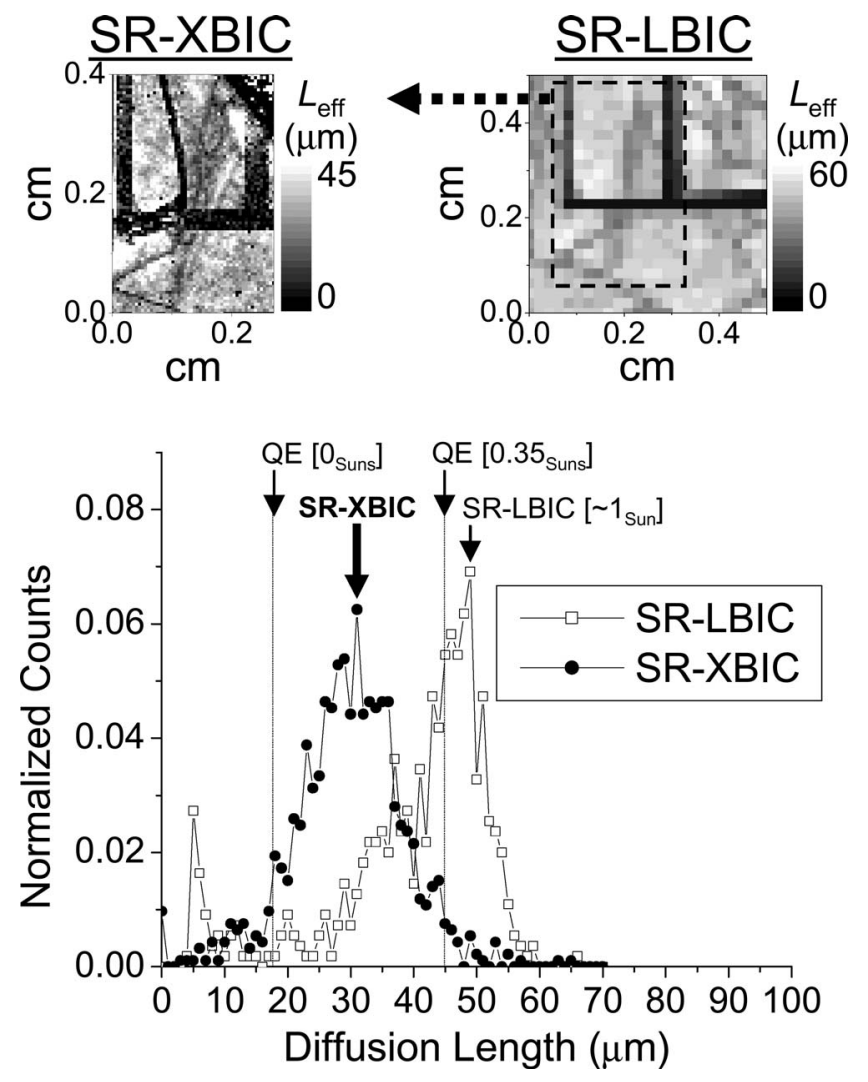

FIG. 2. Comparison between spectrally resolved light/laser beam induced current (SR-LBIC), spectrally resolved x-ray beam induced current (SR$\mathrm{XBIC}$ ), and average diffusion lengths determined from quantum efficiency measurements averaged over the entire wafer with 0 and 0.35 Suns bias illumination. The same features are evident in each map. The slightly lower average minority carrier diffusion length measured in SR-XBIC is believed to be due to the lower minority carrier injection level.

Advanced Light Source Beamline 10.3.2 (a bending magnet source) [Ref. 15].

SR-XBIC was also compared to SR-LBIC, which operates at much higher injection conditions (near 1 Sun), and quantum efficiency measurements averaged over the full wafer area, with variable injection conditions depending on bias lighting, for a solar cell pre-characterized at the Fraunhofer Institute for Solar Energy Systems and the University of Konstanz. Qualitatively, both SR-XBIC and SR-LBIC are very similar, revealing the same recombination-active grain boundaries (Fig. 2). Quantitatively, SR-XBIC and SR-LBIC produce similar peak $L_{\text {eff }}$ values (31 and $49 \mu \mathrm{m}$, respectively), albeit a shift to lower $L_{\text {eff }}$ values is observed for SR-XBIC (Fig. 2). This shift is expected, due to the injection dependence of the minority carrier diffusion length in this particular sample. The average SR-XBIC diffusion length falls within the range of expected values determined by fullwafer quantum efficiency measurements (dotted lines and arrows in Fig. 2), which reveal average diffusion lengths between 18 and $45 \mu \mathrm{m}$ for this sample using low (no bias lighting) and medium-high (0.35 Suns bias lighting) injection conditions, respectively.

When the SR-XBIC technique is combined with the well-established $\mu$-XRF and $\mu$-XAS techniques, one can measure the elemental composition, chemical state, and effect on minority carrier diffusion length of metal-related defects in situ, with micron or submicron spatial resolution. For example, Fig. 3 shows maps of copper and nickel distribu- 

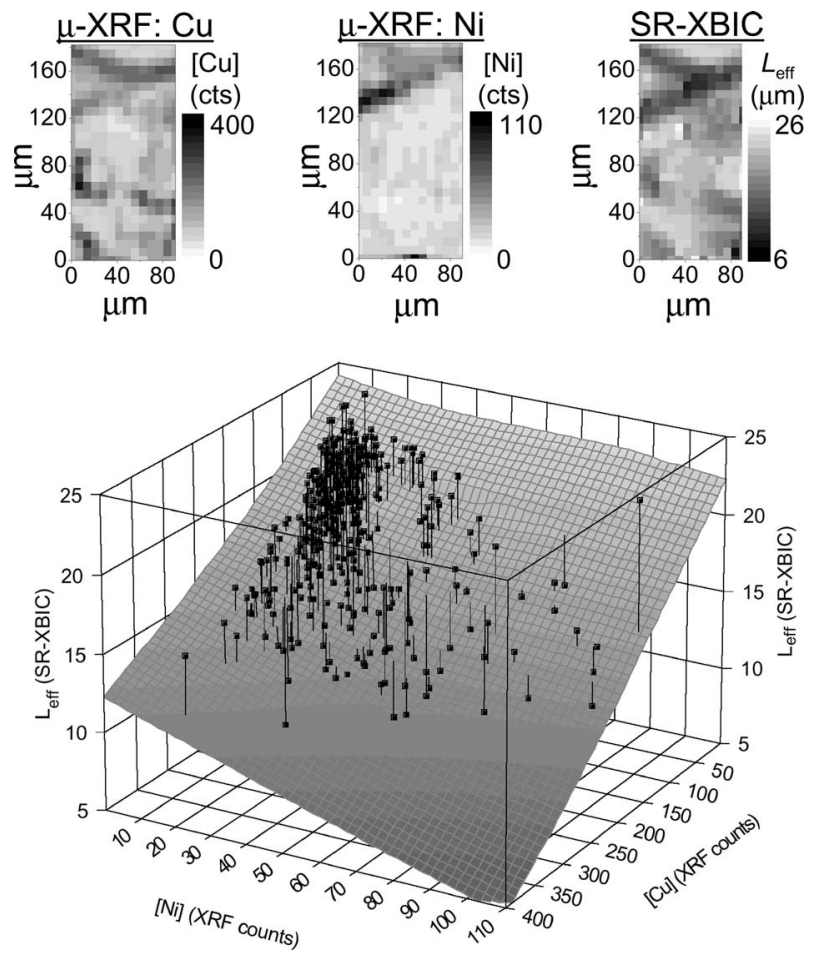

FIG. 3. SR-XBIC data (top right) can be obtained in situ at the $\mu$-XRF beamline, allowing for direct comparisons with metal distributions (top left, middle). The three-dimensional correlation plot demonstrates a strong correlation between increasing metal content and decreasing minority carrier diffusion length.

tions determined by $\mu$-XRF, and minority carrier diffusion length determined by SR-XBIC, of a metal-contaminated multicrystalline float zone (mc-FZ, Ref. 16) sample intentionally contaminated with copper, nickel, and iron in order to achieve a very well-defined impurity distribution. The $\mu$-XAS analyses determine $\mathrm{Cu}$ and $\mathrm{Ni}$ to be in the form of $\mathrm{Cu}_{3} \mathrm{Si}$ and $\mathrm{NiSi}_{2}$, respectively, in agreement with previous results. ${ }^{7} \mathrm{Fe}$ did not form precipitates larger than the detection limit of these experiments, and could not be observed in $\mu$-XRF maps.

Figure 3 demonstrates a clear correlation between local metal concentration and minority carrier diffusion length, and areas with the highest $\mathrm{Cu}$ and $\mathrm{Ni}$ counts have the lowest diffusion lengths. The recombination strengths of $\mathrm{Cu}_{3} \mathrm{Si}$ and $\mathrm{NiSi}_{2}$ appear to be similar, as given by the slopes of the fit in Fig. 3 (note the different scales of the $\mathrm{Cu}$ and $\mathrm{Ni}$ axes). By comparing the SR-XBIC value at the points of maximum $\mathrm{Cu}$ and $\mathrm{Ni}(7 \mu \mathrm{m})$ versus the points with the least of these metals $(25 \mu \mathrm{m})$, one deduces that the presence of $\mathrm{Cu}_{3} \mathrm{Si}$ and $\mathrm{NiSi}_{2}$ precipitates may locally reduce the minority carrier diffusion length by up to $72 \%$ compared to background levels.

When one extrapolates the $\mathrm{Cu}$ and $\mathrm{Ni}$ concentrations to zero in Fig. 3, one determines a low baseline minority carrier diffusion length of approximately $30 \mu \mathrm{m}$. Meanwhile, "clean" mc-FZ samples can obtain local diffusion lengths higher than $100 \mu \mathrm{m}$. The likely explanation of this reduction is iron, present in point defects ${ }^{17}$ or very small clusters. When comparing clean and Fe-contaminated mc-FZ samples, one observes this same effect, while it is not evident in mc-FZ samples contaminated with $\mathrm{Cu}$ only. The low solu- bility and diffusivity of Fe favor the formation of smaller precipitates and point defects, unlike the larger precipitates formed by fast-diffusing and highly soluble $\mathrm{Cu}$ and $\mathrm{Ni}$. The small distances separating neighboring Fe defect clusters increase their effect on device performance.

By combining SR-XBIC and $\mu$-XRF, one achieves a quantitative correlation between minority carrier diffusion length and metal concentrations with micron or submicron spatial resolution. Thus, it becomes possible to decouple the effects of individual metal species and defect types on the minority carrier diffusion length of mc-Si. It was found that submicron-sized $\mathrm{Cu}_{3} \mathrm{Si}$ and $\mathrm{NiSi}_{2}$ precipitates, as well as homogeneous distributions of smaller precipitates and/or point defects, can both significantly reduce the minority carrier diffusion length in mc-Si. In the future, this combination of techniques should make possible the quantitative comparison between different samples, e.g., hydrogen passivated and unpassivated material.

O. F. Vyvenko (SPbSU) is acknowledged for helpful discussions, D. Macdonald (ANU) for lending pre-characterized injection-dependent samples, E. Schäffer (Fraunhofer ISE) for assistance with SR-LBIC measurements. This work was supported by NREL subcontract No. AAT-2-31605-03, with collaboration through the Fraunhofer Institute for Solar Energy Systems (ISE) supported by the AG-Solar project of the government of Northrhein-Westfalia (NRW). The Advanced Light Source is supported by the Director, Office of Science, Office of Basic Energy Sciences, Materials Sciences Division, of the U.S. Department of Energy under Contract No. DE-AC03-76SF00098 at Lawrence Berkeley National Laboratory.

${ }^{1}$ S. A. McHugo, A. C. Thompson, I. Périchaud, and S. Martinuzzi, Appl. Phys. Lett. 72, 3482 (1998).

${ }^{2}$ S. A. McHugo, Appl. Phys. Lett. 71, 1984 (1997).

${ }^{3}$ S. A. McHugo, A. C. Thompson, G. Lamble, C. Flink, and E. R. Weber, Physica B 273, 274, 371 (1999).

${ }^{4}$ T. Buonassisi, A. A. Istratov, M. Heuer, M. Marcus, R. Jonczyk, J. Isenberg, B. Lai, Z. Cai, S. Heald, W. Warta, R. Schindler, G. Willeke, and E. R. Weber, J. Appl. Phys. 97, 074901 (2005).

${ }^{5} \mathrm{H}$. Hieslmair, A. A. Istratov, R. Sachdeva, and E. R. Weber, in Proceedings of the 10th NREL Workshop on Crystalline Silicon Solar Cell Materials and Processes, Copper Mountain, CO, 14-16 August 2000, p. 162

${ }^{6}$ O. F. Vyvenko, T. Buonassisi, A. A. Istratov, H. Hieslmair, A. C. Thompson, R. Schindler, and E. R. Weber, J. Appl. Phys. 91, 3614 (2002).

${ }^{7}$ T. Buonassisi, M. A. Marcus, A. A. Istratov, M. Heuer, T. F. Ciszek, B. Lai, Z. Cai, and E. R. Weber, J. Appl. Phys. 97, 063503 (2005).

${ }^{8}$ T. Buonassisi, O. F. Vyvenko, A. A. Istratov, E. R. Weber, G. Hahn, D. Sontag, J. P. Rakotoniaina, O. Breitenstein, J. Isenberg, and R. Schindler, J. Appl. Phys. 95, 1556 (2004).

${ }^{9}$ W. Warta, Sol. Energy Mater. Sol. Cells 72, 389 (2002).

${ }^{10}$ M. A. Green, Silicon Solar Cells: Advanced Principles \& Practice (University of New South Wales, Sydney, Australia, 1995), Appendix G.

${ }^{11}$ P. A. Basore, IEEE Trans. Electron Devices 37, 337 (1990).

${ }^{12}$ B. L. Henke, E. M. Gullikson, and J. C. Davis, At. Data Nucl. Data Tables 54, 181 (1993).

${ }^{13}$ J. Lagowski, P. Edelman, M. Dexter, and W. Henley, Semicond. Sci. Technol. 7, A185 (1992).

${ }^{14}$ D. K. Schroder, Semiconductor Material and Device Characterization, 2nd ed. (Wiley, New York, 1998).

${ }^{15}$ M. A. Marcus, A. A. MacDowell, R. Celestre, E. Domning, K. Franck, A. Manceau, G. Morrison, T. Miller, H. A. Padmore, and R. E. Sublett, J. Synchrotron Radiat. 11, 239 (2004).

${ }^{16}$ T. F. Ciszek and T. H. Wang, J. Cryst. Growth 237-239, 1685 (2002).

${ }^{17}$ D. H. Macdonald, L. J. Geerligs, and A. Azzizi, J. Appl. Phys. 95, 1021 (2004). 